\title{
Characterization of the Structure of Cation-Doped Bacteriogenic Uranium Oxides using X-Ray Diffraction
}

\author{
Jonathan M. Stahlman \\ Office of Science, Science Undergraduate Laboratory Internship Program
}

Carnegie Mellon University, Pittsburgh

Stanford Linear Accelerator Center

Menlo Park, California

August 25, 2007

Prepared in fulfillment of the requirement of the Office of Science, Department of Energy's Science Undergraduate Laboratory Internship under the direction of Dr. John Bargar and Dr. Eleanor Schofield in the Environmental Remediation Science group at the Stanford Synchrotron Radiation Laboratory at the Stanford Linear Accelerator Center.

Participant:

Signature

Research Advisor:

Signature 


\section{Table of Contents}

Abstract iii

Introduction 1

Materials and Methods $\quad 2$

$\begin{array}{ll}\text { Results } & 6\end{array}$

$\begin{array}{ll}\text { Discussion and Conclusions } & 7\end{array}$

$\begin{array}{ll}\text { Acknowledgments } & 8\end{array}$

$\begin{array}{ll}\text { References } & 9\end{array}$

$\begin{array}{ll}\text { Tables } & 10\end{array}$

$\begin{array}{ll}\text { Figures } & 11\end{array}$ 


\begin{abstract}
Characterization of the Structure of Cation-Doped Bacteriogenic Uranium Oxides using X-Ray Diffraction. JONATHAN M. STAHLMAN (Carnegie Mellon University, Pittsburgh, PA 15289) JOHN BARGAR (Stanford Linear Accelerator Center, Menlo Park, CA 94025)

Remediation of uranium contamination in subsurface groundwater has become imperative as previous research and manufacturing involving radionuclides has led to contamination of groundwater sources. A possible in situ solution for sequestration of uranium is a bacterial process in which Shewanella oneidensis MR-1 reduces the soluble (and thus mobile) $\mathrm{U}(\mathrm{VI})$ oxidation state into the less mobile $\mathrm{UO}_{2}$ crystalline phase. However, the long term stability of the $\mathrm{UO}_{2}$ compound must be studied as oxidative conditions could return it back into the U(VI) state. Incorporation of other cations into the structure during manufacture of the $\mathrm{UO}_{2}$ could alter the dissolution behavior. A wide angle x-ray scattering (WAXS) experiment was performed to determine whether or not calcium, manganese, and magnesium are incorporated into this structure. If so, the substituted atoms would cause a contraction or expansion in the lattice because of their differing size, causing the lattice constant to be altered. After several stages of data reduction, the WAXS diffraction peaks were fit using the Le Bail fit method in order to determine the lattice constant. Initial results suggest that there may be incorporation of manganese into the $\mathrm{UO}_{2}$ structure due to a $.03 \AA$ decrease in lattice constant, but more data is needed to confirm this. The calcium and magnesium doped samples showed little to no change in the lattice constant, indicating no significant incorporation into the structure. Most importantly, this experiment revealed an artifact of the cleaning process used to remove the bacteria from the sample. It appears the $\mathrm{NaOH}$ used to clean the samples is contracting the lattice also by $\sim .03 \AA$, but no physical explanation is offered as of yet.
\end{abstract}




\section{Introduction}

Research in the remediation of uranium contamination in subsurface groundwater is imperative as the Department of Energy (DOE) has many sites in which previous research and manufacturing involving radionuclides has led to contamination of local groundwater sources. The total estimated volume of groundwater contaminated with radionuclides is over 1.7 trillion gallons in over 5,700 plumes [1]. Because of this enormous reserve, leaching of uranium into groundwater could pose a continuing environmental and health risk for countless years.

Steps have been taken to combat this problem. Due to the enormity of the contamination, extraction would prove not only to be prohibitively expensive but almost impossible in practice [1]. Thus, a search for an efficient method of in situ sequestration has been undertaken. A possible solution for uranium sequestration is the stimulation of Shewanella oneidensis MR-1, a bacterial strain which can reduce the soluble (and thus mobile) U(VI) oxidation state into the less mobile U(IV) state[2]. Once in this state, the uranium will precipitate out as nanoparticulate crystalline $\mathrm{UO}_{2}$. Stimulation of this process is easily accomplished, but the long term stability of this solution is unknown. If the bacteriogenic $\mathrm{UO}_{2}$ were to be exposed to oxidative conditions, it could then return to its mobile $\mathrm{U}(\mathrm{VI})$ state and once more pose a risk.

Along with collaborators, the Environmental Remediation Science group at the Stanford Synchrotron Radiation Laboratory (SSRL) is investigating the long term stability of the $\mathrm{UO}_{2}$ compound. Because the actual sequestration of the uranium will occur in the subsurface, a thorough study of the environmental effects must be done. The goal of this project is to determine if the presence of cations, such as $\mathrm{Ca}^{2+}$, during the bacterial process alters the basic structure of the $\mathrm{UO}_{2}$ crystal, leading to changes in the solubility of the compound. 
Samples for the study are prepared at the Ecole Polytechnique Federale de Lausanne (EPFL). The synchrotron facilities at SSRL allow for characterization of both the long range and local structure of the bacteriogenic $\mathrm{UO}_{2}$ compounds using a variety of $\mathrm{x}$-ray scattering and spectroscopy techniques. Simultaneously, collaborators at Washington University in St. Louis are analyzing the solubility and dissolution rates of the various compounds. Putting these results together produces an overall understanding of the structure and behavior of bacteriogenic $\mathrm{UO}_{2}$ in subsurface conditions.

In this paper, we will analyze the results of a wide angle x-ray scattering (WAXS) experiment on various cation-doped bacteriogenic $\mathrm{UO}_{2}$ compounds. Specifically, we analyze samples in which $\mathrm{Ca}, \mathrm{Mn}$, and $\mathrm{Mg}$ cations have been added. These cations were chosen as they are normally present in groundwater. After several stages of data reduction, we are able to extract the lattice constant of the $\mathrm{UO}_{2}$. To determine the lattice constant, we use the technique of Le Bail fitting, a derivative of the Rietveld Refinement method. If the cations had been incorporated into the crystal, we predict that the crystal lattice should contract or expand due to the difference in atomic sizes of the cations compared to the uranium, resulting in a measurably different lattice constant.

\section{Materials \& Methods}

An array of uranium oxide samples were prepared by collaborators at EPFL. Each was prepared under a unique set of conditions, which include the concentration of dopant, $\mathrm{pH}$, and cleaning agent used. A summary of these samples and their characteristics is seen in Tables 1 and 2. A cleaning agent is used to remove the bacterial cells from the samples in order to reduce background noise in the diffraction patterns. 
Once at SSRL, the wet samples were loaded into $0.2 \mathrm{~mm}$ glass capillary tubes in an anaerobic chamber. The sample capillaries were then mounted in sealed containers with a constant flow of nitrogen in order to preserve the anaerobic conditions. Sealed kapton windows were added to the containers in order for the x-ray beam to pass through the container without adding background noise.

A diagram of the experimental setup can be seen in Figure 1. Diffraction measurements were taken at beamline 7-2 at the SSRL facility using a Huber 6-circle diffractometer. Intensity measurements were taken using a vortex detector before and after the beam strikes the sample. The second detector, labeled $\mathrm{I}_{1}$, scanned over a range of $\mathrm{Q}$ values from $0.8 \AA^{-1}$ to $14.5 \AA^{-1}$ in .02 increments. As in most diffraction experiments, $Q$ is defined as :

$$
Q=\frac{4 \pi \sin (2 \theta / 2)}{\lambda}
$$

where $\lambda$ is $x$-ray wavelength and $2 \theta$ is the angle between the diffracted beam and the unscattered beam. This was all done with a monochromatic x-ray beam of $0.7696 \AA$. The $\mathrm{UO}_{2}$ is nanoparticulate, so the experiment is one of powder diffraction. Thus, the diffraction pattern will be cylindrically symmetric around the axis of the beam. Therefore, it was only necessary to scan over one slice of this diffraction pattern in $2 \theta$ space.

At each Q value, the energy spectrum of the signal was recorded. This will later be used to subtract out the inelastically scattered $x$-rays. Three to four scans over the $2 \theta$ range were taken of each sample and then directly summed together. LaB6, a diffraction standard, was used to determine the wavelength of the x-ray beam. In addition to our samples, an empty capillary was also scanned for later background subtraction. 
First, the inelastic scattered x-ray signal (or Compton scattered) was subtracted from the data. The Compton scattered x-rays must be subtracted because the Le Bail analysis does not take this phenomenon into account. The wavelength of the Compton scattered x-rays goes as:

$$
\lambda^{\prime}=\lambda+\frac{h}{m_{e} c}(1-\cos (2 \theta / 2))
$$

where $\lambda^{\prime}$ is the scattered wavelength and $\mathrm{h} / \mathrm{m}_{\mathrm{e}} \mathrm{c}$ is known as the Compton wavelength, equal to $2.43 \times 10^{-2} \AA$. Because the Compton wavelength is small relative to the initial x-ray wavelength, there is not much difference in wavelength between Compton scattered and elastic scattered xrays at small $2 \theta$. Therefore, separating the elastic signal from the Compton signal at this angle will be quite difficult. However, for our setup, above $2 \theta=80^{\circ}$, the two peaks are easily discernible, as seen in Figure 2.

To separate the elastic signal from the Compton signal, the energy spectrum scans with Q $>10.78$ are first fit with a pair of Gaussian peaks, using the peak center, peak width, and peak area as the parameters for the fit. This initial fit gave three pieces of information about the two peaks. The elastic peak energy theoretically should not change, so the peak center was averaged from this first fit. As well, both peak widths appeared to be constant, so these were averaged. These three average values were then used as constants in fits over all Q and more accurate fits were thus achieved at lower $\mathrm{Q}$ values. The area of the elastic peak was then used to determine the ratio of elastic counts to total counts for each $\mathrm{Q}$ value. This ratio was then used to scale the summed data to extract the elastic signal.

Background subtraction of the capillary reflections was accomplished using the software package XRD-BS [4]. Scaling of the capillary data for subtraction from the diffraction data was 
done by visual inspection for each sample for accuracy. In addition, the program can also account for absorption corrections for cylindrical geometries given a value of $\mu \mathrm{L}$, the absorption coefficient multiplied by the total length traversed by the x-ray beam. This quantity depends on the physical makeup of the sample as well as its geometry. It appears in the equation:

$$
I=I_{0} e^{-\mu L}
$$

where $\mathrm{I}$ is the observed intensity and $\mathrm{I}_{0}$ is the initial intensity. $\mu \mathrm{L}$ was estimated experimentally by measuring the intensity of the beam with and without the sample in the path of the beam. The negative natural $\log$ of the ratio $\mathrm{I} / \mathrm{I}_{0}$ then gives a value of $\mu \mathrm{L}$ for each sample. Values of $\mu \mathrm{L}$ for our samples ranged from 0.3 to 1.5 .

The diffraction data was then analyzed using the General Structure Analysis System (GSAS) software package along with EXPGUI [3,4]. Specifically, we used the Le Bail fitting method, a derivative of the Rietveld Refinement. Unlike a Rietveld Refinement, a Le Bail fit takes the observed intensities as arbitrary unknowns and uses the space group, lattice constants, and other parameters to fit the diffraction data without taking into consideration the locations of the atoms within the unit cell. This method is ideal for extracting the lattice constant, though some care must be taken in assuring the fit makes physical sense[6]. $\mathrm{UO}_{2}$ exists in a facecentered cubic structure, thus making the analysis much simpler, as only one lattice constant must be determined from the fit.

A pseudo Voigt profile was chosen to model the peaks. This profile mixes Gaussian and Lorentzian functions to fit the diffraction peak. Most importantly, the profile function is used to describe the broadening of the peaks. This may be caused by several things: microstrain, experiment instrumentation, and particle size. Examining our LaB6 calibration data, we see very 
little broadening (compared to the raw data), so we can assume that instrumental broadening is negligible. Thus, only two profile parameters were used to fit the data: a Lorentzian particle broadening term and a Gaussian strain broadening term. This combination tended to give the best fits. In addition, several parameters to model a broad background and a zero offset in Q space were added in order to further improve the Le Bail fits. A good fit is determined by examining the value of reduced $\mathrm{X}^{2}$ for the fit.

After a good fit is attained, the parameters of the fit provide the value of the lattice constant. In addition, GSAS provides an estimate of the uncertainty on the given value of the lattice constant. Theoretically, the particle size could also be inferred from the Le Bail fits. However, this is not always the most reliable method as peak broadening could be caused by microstrain or particle size. Thus, separation of the two with a Le Bail fit can be somewhat unreliable [6].

\section{Results}

A plot of the raw data can be seen in Figure 3. The first nine peaks have been labeled with the appropriate Miller indices. Notice the very broad peaks. This is due to the nanoparticulate nature of the $\mathrm{UO}_{2}$ crystals.

Figure 4 plots peak center as a function of $\mathrm{Q}$ from the Compton fitting before and after the constants have been determined for one of the samples. In addition, the lighter colors represent the width of the peak. Figure 5 shows a plot of the diffraction data before and after Compton subtraction. A sample background subtraction can be seen in Figure 6.

A sample Le Bail fit is shown in Figure 7. Table 1 presents the lattice constants of all of the doped samples while Table 2 summarizes the results of the undoped samples. All uncertainties were generated using GSAS. 


\section{Discussion}

Examining Figure 4, this shows that fixing the three variables mentioned earlier results in much better fitting for later Compton subtraction. Theoretically, the Compton peak center should intersect the elastic center at $\mathrm{Q}=0$. This does not happen with our fitting, but the correction is still a good approximation. In addition, the Compton subtraction isn't entirely vital to extracting the lattice constants using a Le Bail fit. This occurs because the value of the lattice constant is adjusted according to the location in Q space of the peak and does not rely heavily on the intensity or width of the peak. The Compton correction is a broad spectrum effect and does not significantly change the shape of the diffraction data (Figure 5). Still, it is important to do this correction in anticipation of a more rigorous analysis, such as a Rietveld Refinement, which is planned for the future.

The background subtraction of the capillary signal is also shown to be adequate. Though minor, this is still an important consideration and significantly improved our data.

The results of the Le Bail fitting are somewhat surprising. The $\mathrm{Ca}^{2+}$ cation is slightly larger than the $\mathrm{U}^{4+}$, while $\mathrm{Mg}^{2+}$ is slightly smaller. However, examining Table 1 , we see that the calcium and magnesium showed little difference in their lattice constants. Also, we were only able to process one sample for each of these dopants. More samples would confirm whether a real trend actually exists. If the lattice constants truly haven't changed, then we must infer that these elements are not being incorporated into the $\mathrm{UO}_{2}$ structure.

The manganese samples gave mixed results. The $.1 \mathrm{mM}$ sample and the $5 \mathrm{mM}$ sample both showed lattice contractions of $\sim .03 \AA$. $\mathrm{Mn}^{2+}$ has an atomic radius of $.93 \AA$ while $\mathrm{U}^{4+}$ has a radius of $1.0 \AA$. Thus, the lattice contraction we measure for these two samples is of the right order of magnitude to be consistent with our prediction that manganese is being substituted into 
the structure in place of the uranium. However, the $1 \mathrm{mM}$ sample showed no lattice contraction. This is quite puzzling as this completely goes against the trend. To clarify this, we hope to reexamine the data and possibly repeat the experiment for better confirmation.

One very unpredicted result was the result presented in Table 2. Here, we discover that the cleaning method employing $\mathrm{NaOH}$ seems to be changing the lattice constant of the $\mathrm{UO}_{2}$. Physically, we are unable at the moment to explain the reasoning why this cleaning process would be changing the $\mathrm{UO}_{2}$ structure. This is quite unexpected and must be taken care of immediately before any other experiments can be done. The Lysozyme cleaning does not seem to have this effect on the structure, so it may become the cleaning method of choice.

We have analyzed the results of a WAXS experiment on cation doped bacteriogenic $\mathrm{UO}_{2}$ and found several interesting features. The Mn doped samples show a likely lattice contraction, but mixed results have caused doubt as to whether this is true or not. More importantly we have discovered that our cleaning method is affecting the structure of that which we are studying. Thus, we must change our methods of sample preparation. Future work will include a more rigorous analysis of the data, most likely using the Rietveld Refinement method. This analysis will produce information about the structure such as atomic positions and particle size. Overall, this experiment has helped to characterize the structure of doped bacteriogenic $\mathrm{UO}_{2}$.

\section{Acknowledgments}

We would like to acknowledge the Department of Energy and the SULI program for funding this research. We would also like to thank Apurva Mehta as an invaluable source of information during this research. Finally, we would like to express our gratitude to our mentors John Bargar and Eleanor Schofield for all of their guidance and help in completing this research. 


\section{References}

[1] U.S. Department of Energy. Office of Science. Office of Biological Research.

Bioremediation of Metal and Radionuclides... What It is and How It Works. 2nd. 2003.

[2] Chongxuan Liu, Yuri A. Gorby, John M. Zachara, Jim K. Fredrickson, Christopher F. Brown, " Reduction kinetics of Fe(III), Co(III), U(VI), Cr(VI), and Tc(VII) in cultures of dissimilatory metal-reducing bacteria," Biotechnology and Bioengineering, vol. 80, no. 6, pp. 637-649, 2002.

[3] A.C. Larson and R.B. Von Dreele, "General Structure Analysis System (GSAS)", Los Alamos National Laboratory Report LAUR 86-748 (2000).

[4] B. H. Toby, EXPGUI, a graphical user interface for GSAS, Journal of Applied Crystallography, vol. 34, 210-213, 2001.

[5] S. Webb. XRD-BS, Stanford Linear Accelerator Center. (2006).

[6] D. Balzar, et al., " Size-strain line-broadening analysis of the ceria round-robin sample ," Journal of Applied Crystallography, vol. 37, pp. 911-924, 2004.

[7] "A Profile Refinement Method for Nuclear and Magnetic Structures." Rietveld, H.M., Journal of Applied Crystallography, vol. 2, pp. 65-71, 1969. 
Fit Lattice Constants of Doped Bacteriogenic $\mathrm{UO}_{2}$ Samples

\begin{tabular}{|l|c|c|c|}
\hline & Calcium $(\mathbf{p H = 6 . 0})$ & Magnesium $(\mathbf{p H = 8 . 0})$ & Magnesium $(\mathbf{p H}=\mathbf{6 . 3})$ \\
\hline Undoped & $5.4437 \pm .0029 \AA$ & $5.4307 \pm .0016 \AA$ & $5.4331 \pm .0016 \AA$ \\
\hline $.1 \mathrm{mM}$ & - & - & $5.3956 \pm .0061 \AA$ \\
\hline $1 \mathrm{mM}$ & - & - & $5.4387 \pm .0016 \AA$ \\
\hline $5 \mathrm{mM}$ & - & - & $5.4018 \pm .0022 \AA$ \\
\hline $10 \mathrm{mM}$ & $5.4353 \pm .0022 \AA$ & $5.4405 \pm .0045 \AA$ & - \\
\hline
\end{tabular}

Table 1. Summary of Doped Samples - All were cleaned using $\mathrm{NaOH}$

\begin{tabular}{|l|l|l|l|}
\hline \multicolumn{5}{|c|}{ Fit Lattice Constants of Undoped Bacteriogenic UO 2 Samples } \\
\hline Cleaning Method & \multicolumn{1}{|c|}{ None } & NaOH & Lysozyme \\
\hline $\mathrm{pH}=8$ & $5.4753 \pm .0025 \AA$ & $5.4307 \pm .0016 \AA$ & $5.4702 \pm .0016 \AA$ \\
\hline $\mathrm{pH}=6.3$ & - & $5.4331 \pm .0016 \AA$ & $5.4643 \pm .0017 \AA$ \\
\hline $\mathrm{pH}=6$ & - & $5.4437 \pm .0029 \AA$ & - \\
\hline
\end{tabular}

Table 2. Summary of Undoped Samples 


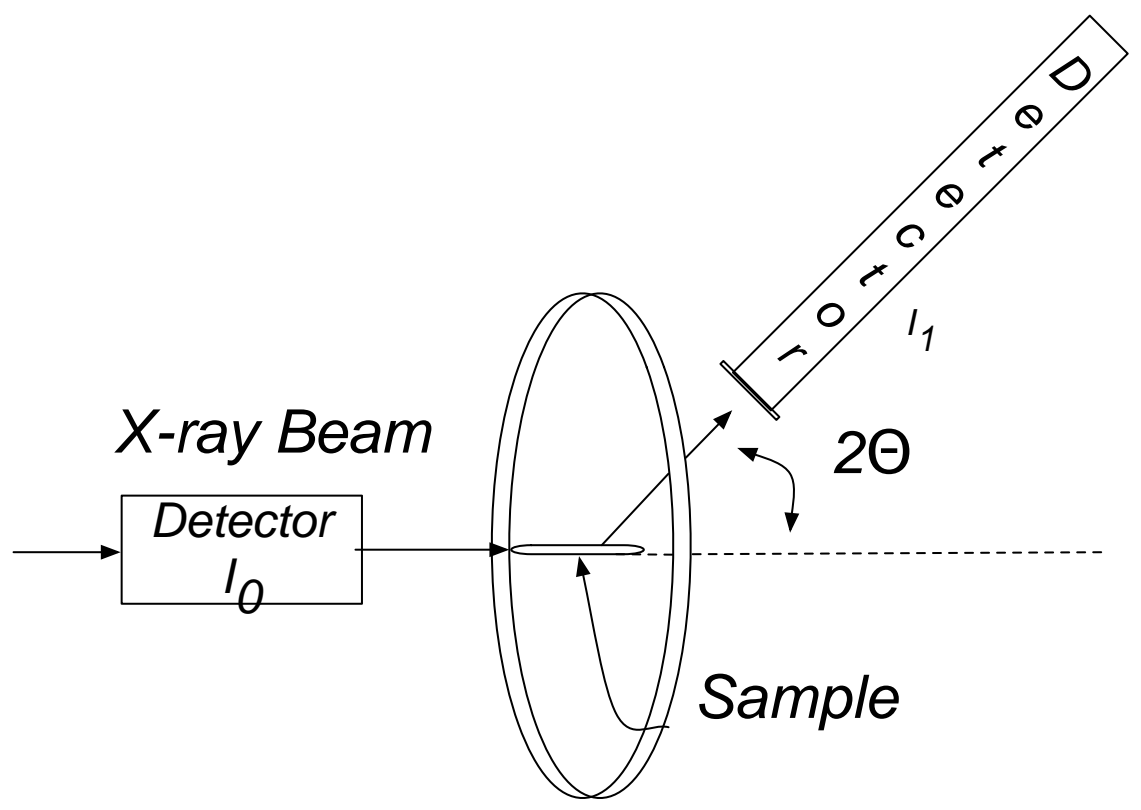

Figure 1. Diagram of Experimental Setup

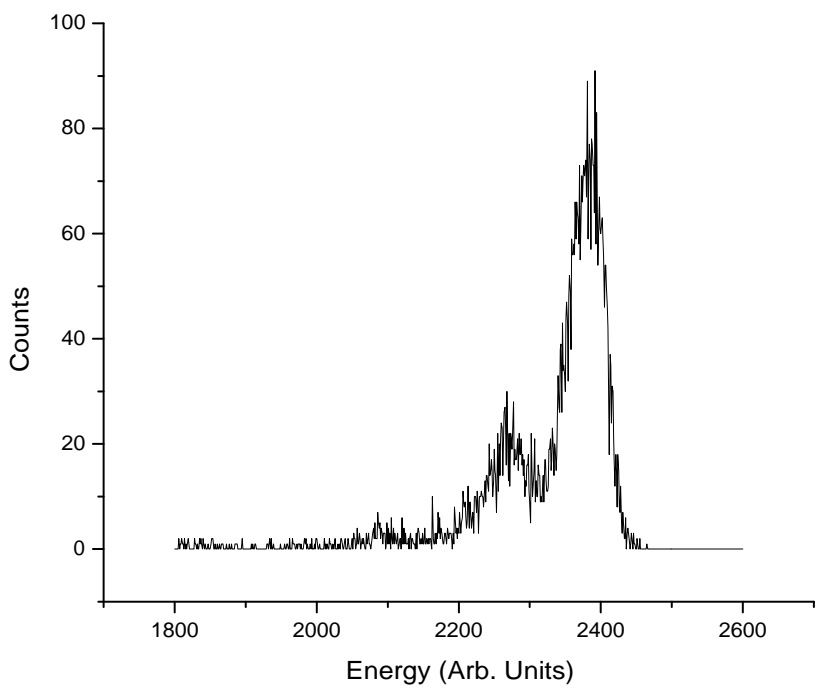

Figure 2. Energy Spectrum at High Q 


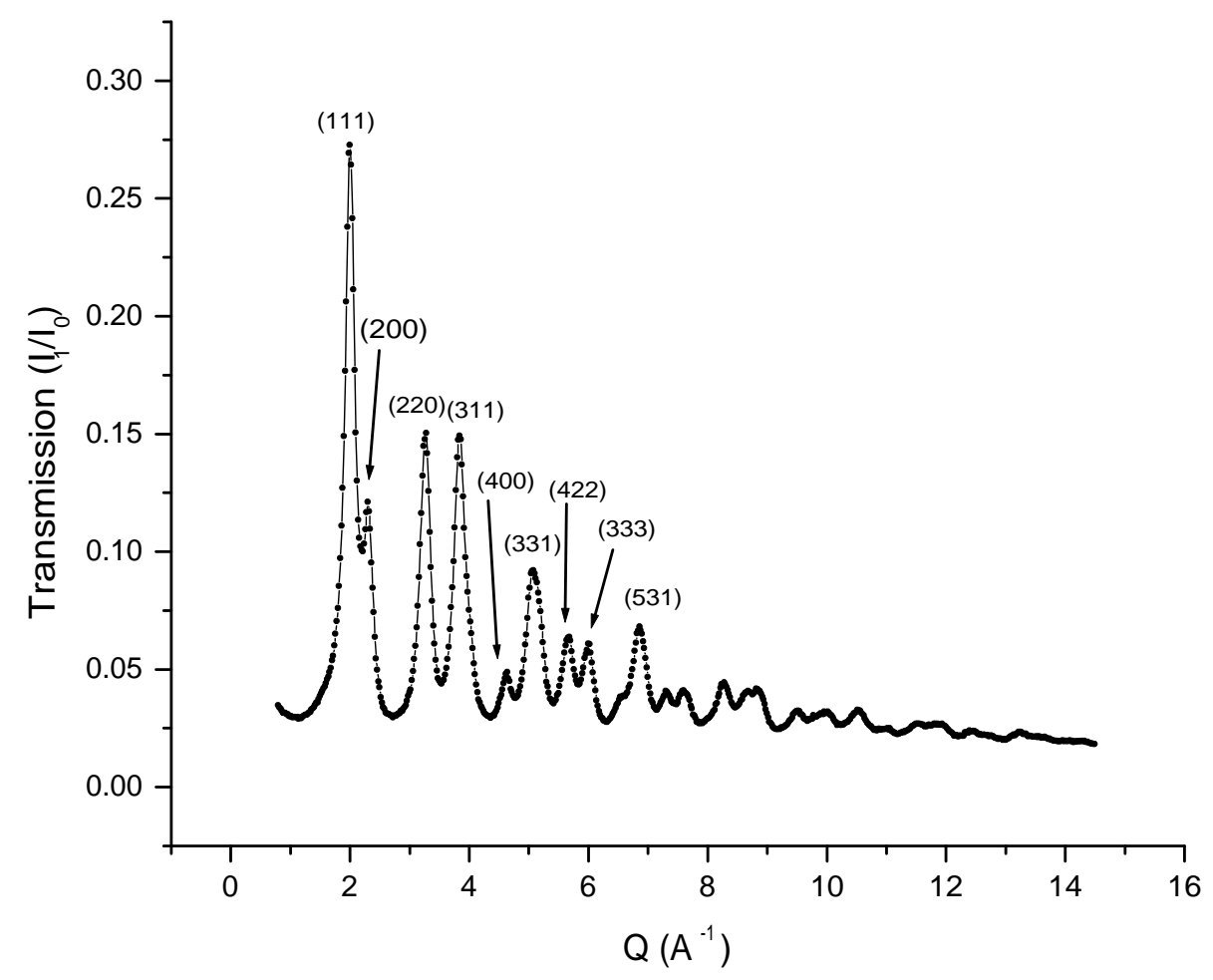

Figure 3. Raw data with first nine peaks labeled with Miller Indices 

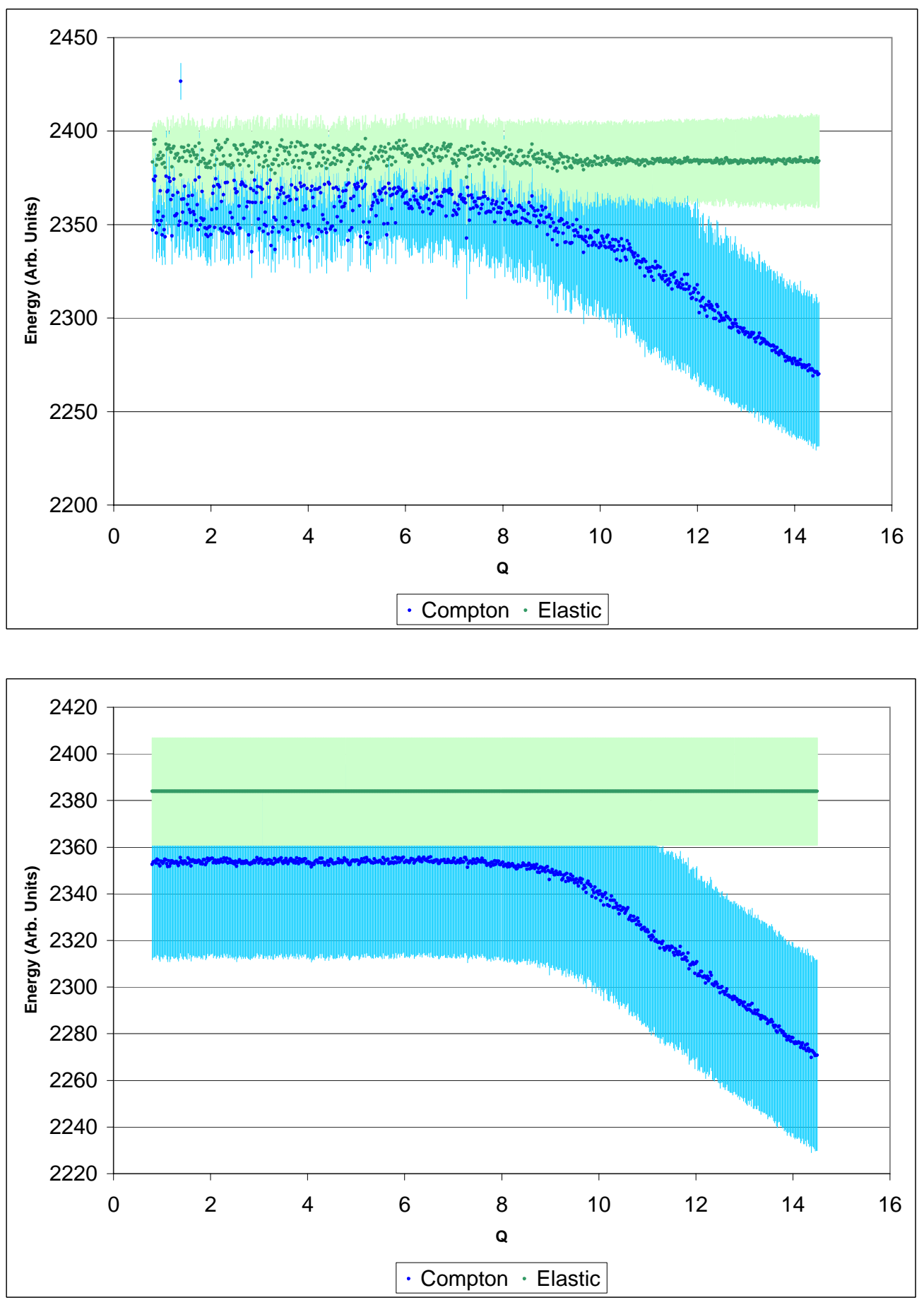

Figure 4. Top - Initial Compton Fittting

Bottom- Final Compton Fit - Elastic peak position and width, and Compton width held constant 


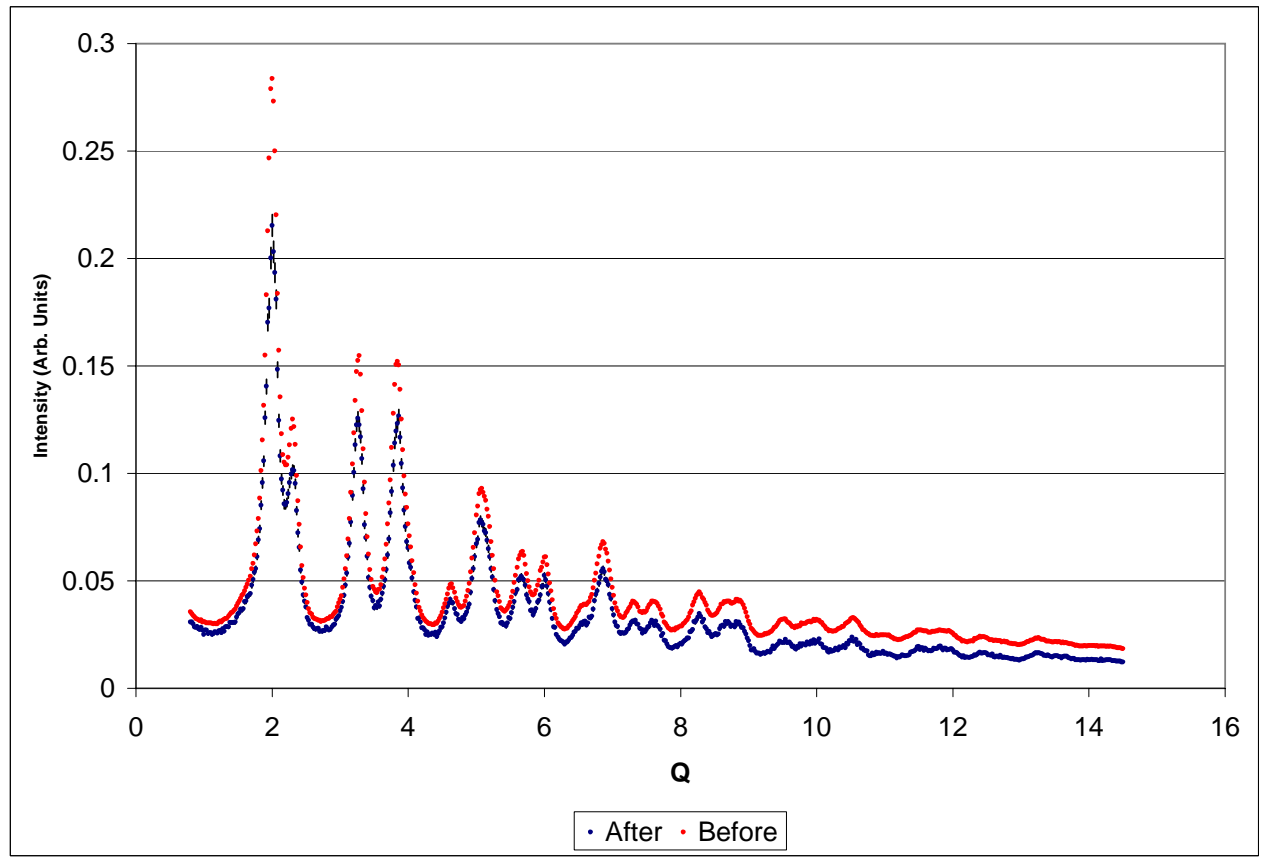

Figure 5. Sample Compton Subtraction 


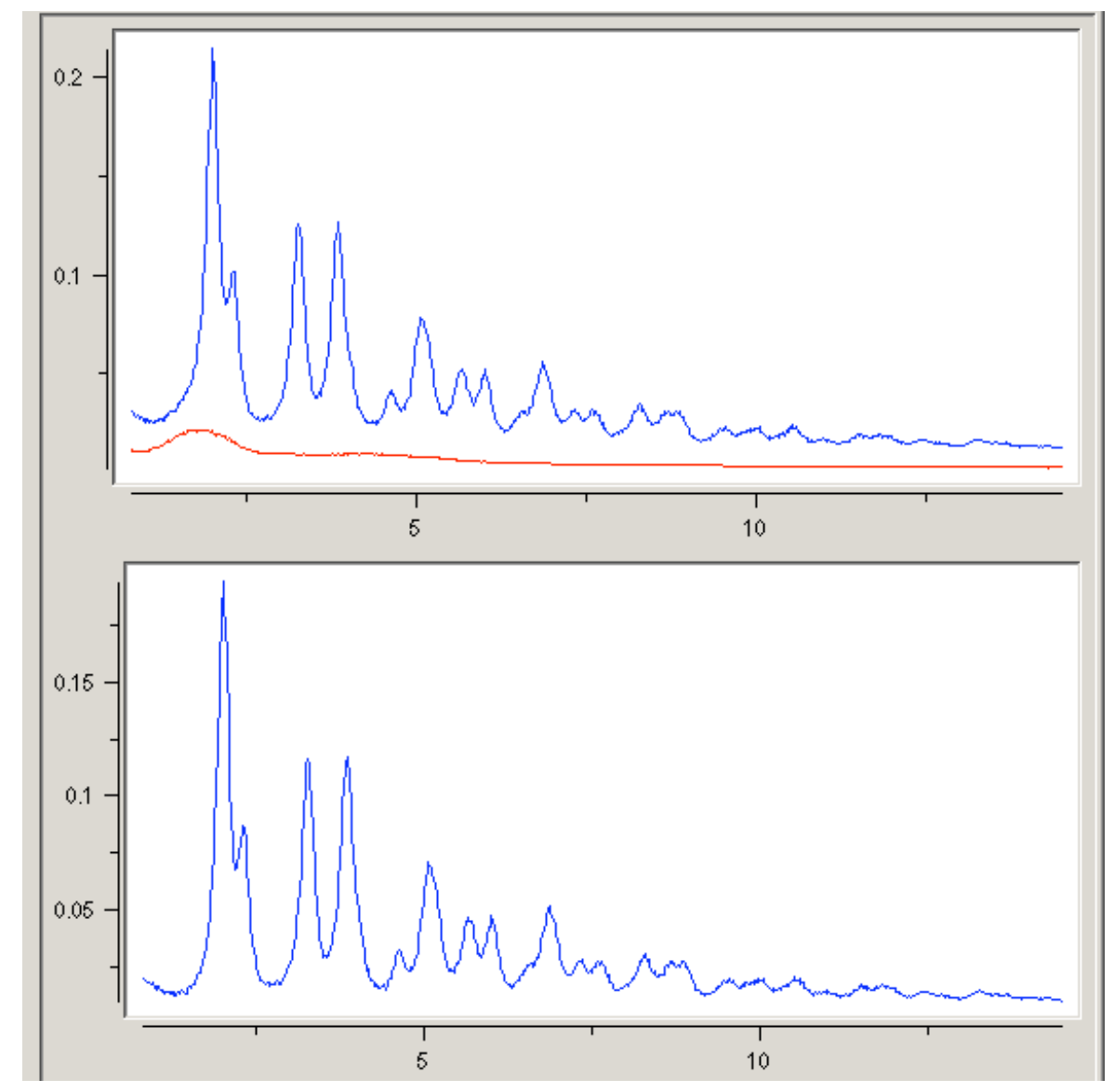

Figure 6. Background Subtraction of Capillary Signal.

Blue Line $=$ Sample Data $;$ Red Line $=$ Capillary Data

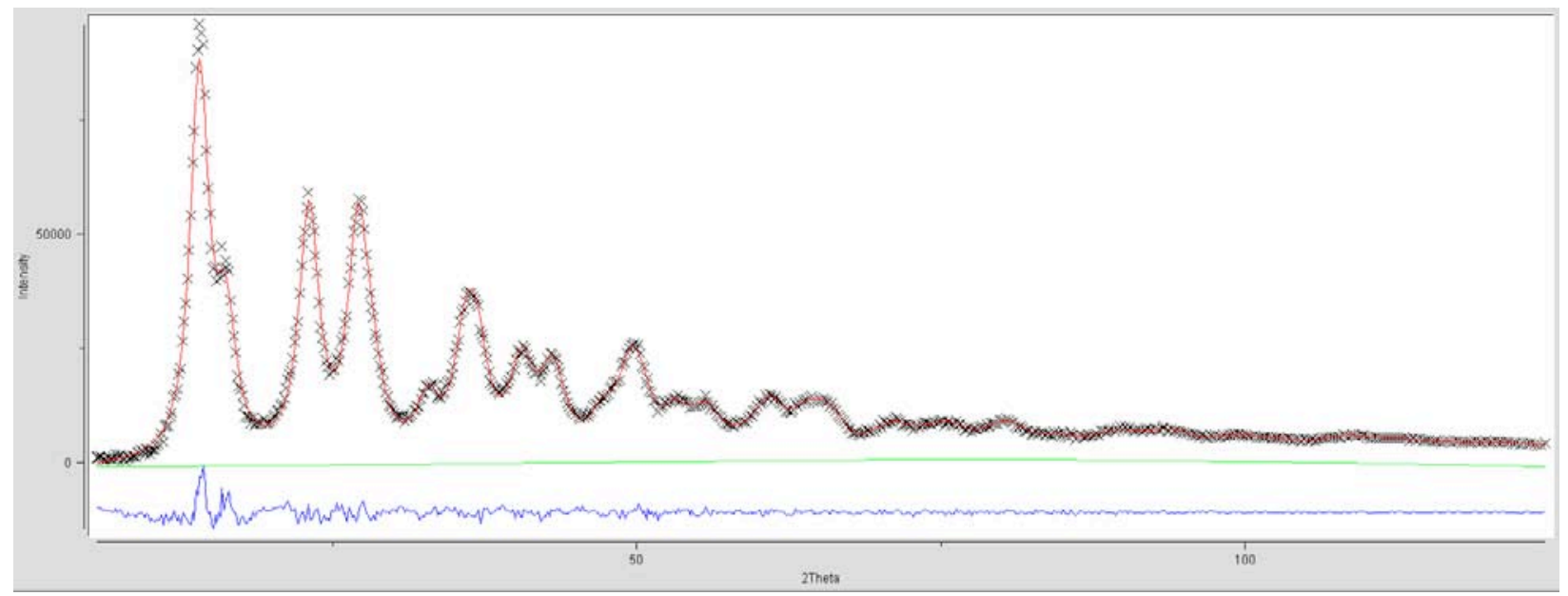

Figure 7. Sample Le Bail Fit

Red Line - Fit Line; Green Line - Background; Blue Line - Error in Fitting 